\title{
Los partidos políticos abiertos: aplicación de los principios del gobierno abierto a las formaciones políticas españolas ${ }^{1}$
}

\section{Open political parties: applying the principles of Open Government to Spanish political affiliations}

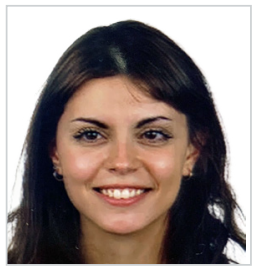

María Díez-Garrido. Investigadora en Comunicación por la Universidad de Valladolid. Es graduada en Periodismo y cursó el Máster en Investigación como agente histórico-social en la misma universidad. Forma parte del equipo de investigación del proyecto $\mathrm{I}+\mathrm{D}+\mathrm{i}$ "Estrategias, agendas y discursos en las cibercampañas electorales: medios de comunicación y ciudadanos" de la Universidad de Valencia (CSO2016-77331-C2-1-R) y de la Red de Excelencia en Periodismo Digital y Convergencia Mediática (CSO2016-81882-REDT), financiados por el Ministerio de Economía y Competitividad. Ha colaborado con el Observatorio de Participación Ciudadana, que forma parte de los compromisos del III Plan de Acción de Gobierno abierto de España, coordinado por el Centro de Estudios Políticos y Constitucionales. Sus trabajos de investigación se centran en la transparencia, el Gobierno abierto y comunicación digital.

Universidad de Valladolid, España

maria.diez.garrido@uva.es

ORCID: 0000-0002-5430-7708

Recibido: 23/03/2020 - Aceptado: 14/10/2020

Resumen:

El Gobierno abierto ha experimentado un fuerte auge en el escenario político y en los estudios académicos. Este artículo reflexiona sobre la adaptación de los pilares del Gobierno abierto (transparencia, participación y colaboración) a los partidos políticos. Estas formaciones en España sufren una gran desconfianza ciudadana, que se suma al alto porcentaje de aportaciones públicas en su financiación. Todo ello hace necesario desarrollar el concepto de partidos políticos abiertos. El estudio parte de una aproximación conceptual, realizada con la metodología Delphi, en el que 20 expertos de los campos de la Comunicación, el Derecho y las Ciencias Políticas han aportado sus conocimientos en dos rondas de cuestionarios. Los resultados sirvieron para redactar una Guía de buenas prácticas para partidos políticos abiertos, que posteriormente se ha utilizado para evaluar a las formaciones. El análisis muestra que los partidos aún deben desarrollar los pilares, especialmente su implicación e interacción de la ciudadanía.

Palabras clave:

Transparencia; partidos políticos abiertos; gobierno abierto; participación; colaboración.

Received: 23/03/2020 - Accepted: 14/10/2020

\section{Abstract:}

Open government has experienced strong development in the political arena as well as in academic studies. This article explores the possibility of adapting the foundations of open government (transparency, participation and collaboration) to political parties. In Spain, such parties have experienced great mistrust from citizens, which goes hand in hand with the high level of public contributions in their financing. These factors have made it necessary to develop the concept of open political parties. This study is based on a conceptual approach using the Delphi methodology in which 20 experts from the fields of Communication, Law and Political Science have contributed their knowledge in two rounds of questionnaires. The results were used to draft a Guide to Best Practice for Open Political Parties, which was used later to evaluate these organisations. The analysis shows that parties still have not developed the foundations mentioned above, especially with regard to their involvement and interaction with citizens.

\section{Keywords:}

Transparency; open political parties; open government; participation; collaboration.

1 Esta investigación se ha elaborado en el marco del proyecto $\mathrm{I}+\mathrm{D}+\mathrm{i}$ "Estrategias, agendas y discursos en las cibercampañas electorales: medios de comunicación y ciudadanos" de la Universidad de Valencia (CSO2016-77331-C2-1-R), financiado por el Ministerio de Economía y Competitividad.

\section{Cómo citar este artículo:}

Díez-Garrido, M. (2020). Los partidos políticos abiertos: aplicación de los principios del gobierno abierto a las formaciones políticas españolas. Doxa Comunicación, 31, pp. 63-86. 


\section{Introducción}

El Gobierno abierto tiene la capacidad de mejorar los servicios públicos, empoderar a la ciudadanía y desarrollar medidas más eficientes (Abu-Shamab, 2015). Es por ello que académicos, políticos y ciudadanos confían en las estrategias de apertura de las instituciones, que deben estar basadas en la transparencia, la participación y la colaboración.

El Gobierno abierto se ha estudiado desde un punto de vista positivo para la administración y se ha centrado fundamentalmente en su aplicación en gobiernos estatales y locales (Ruijer et al., 2019; Bvuma y Joseph, 2019). También ha habido investigaciones sobre la aplicación de estos pilares en el caso de los medios de comunicación (Porlezza y Splendore, 2019) y desde la perspectiva ciudadana (Wirtz et al., 2019). Sin embargo, no se han hallado referencias desde el punto de vista de los partidos políticos.

Este trabajo plantea la posibilidad de extender este tipo de iniciativas a los partidos políticos, no solo a las instituciones públicas, de forma que estos fomenten los principios del Gobierno abierto con el objetivo de acercarse a la ciudadanía y volverse más eficientes, más transparentes, tomar decisiones legitimadas y comunicar sus políticas de una manera más eficaz.

Por ello, en esta investigación se quiere definir el concepto de partidos políticos abiertos y evaluar su grado de ejecución en España. Ya existen diversas organizaciones que periódicamente realizan mediciones de la transparencia de los partidos políticos, como destaca Sierra (2018), casi todas con mecanismos similares, que consisten en la comprobación del cumplimiento de diversos indicadores y aplicando criterios desde el punto de vista descriptivo y cuantitativo. La definición de partidos políticos abiertos hace necesaria una nueva metodología que evalúe el grado de apertura de las formaciones políticas españolas.

\subsection{El paradigma del gobierno abierto}

El interés por el Gobierno abierto comenzó ya en el siglo pasado, pero es en la última década cuando ha recibido una mayor atención. Este auge recibe diversas explicaciones: por un lado, cuando Barack Obama asumió en 2009 la presidencia de Estados Unidos, pronunció el conocido Memorando por la Transparencia y el Gobierno abierto, en el que se comprometió a fomentar el acceso a la información, así como la participación y la colaboración ciudadanas. Por otro lado, las administraciones han comprendido y asumido los cambios tecnológicos y están adaptando sus servicios a estos (Ruvalcaba-Gómez, Sandoval-Almazán, Criado y Valle-Cruz, 2019) a través de la puesta en marcha de portales de transparencia y Gobierno abierto y diferentes planes y medidas. La lucha contra la corrupción también ha incentivado esta tendencia hacia el Gobierno abierto, ya que, aunque no sea la solución, se considera que la transparencia desincentiva este tipo de fraudes (Attard, Orlandi, Scerri y Auer, 2015).

En el campo académico, este interés también ha sido notable, con una amplia cantidad de publicaciones de artículos científicos, libros y comunicaciones en torno al Gobierno abierto, la transparencia y el e-gobierno en todo el mundo, especialmente centrados en los países anglosajones. 
En la práctica, la apertura de las administraciones se ha traducido en la publicación de documentos y conjuntos de datos en Internet. Pero, según explican Meijer, Curtin y Hillebrandt (2012), esto menosprecia el verdadero valor del Gobierno abierto, que no solo afecta a la divulgación de información, sino también a las relaciones con la ciudadanía y estas no se deben descuidar. Así, el Gobierno abierto va más allá que la transparencia y es importante diferenciarlo del e-gobierno, que hacer referencia a la adaptación de las instituciones a las nuevas tecnologías. El Gobierno abierto, en cambio, está ligado a la regeneración de la democracia y está basado en los principios de la transparencia, la participación y la colaboración (Abu-Shamab, 2015). Según McDermott (2012), un Gobierno abierto debe:

- Ser transparente y ofrecer información a la ciudadanía sobre las medidas que se están tomando, desde las instituciones y promover la rendición de cuentas. Se debe diferenciar, además, entre la transparencia activa (Rubio, 2011), que supone la publicación voluntaria de datos, y la transparencia pasiva, que supone la difusión de información tras una solicitud y que debe regularse adecuadamente (Grimmelikhuijsen, 2012).

- Fomentar la participación ciudadana, pues las administraciones pueden nutrirse del conocimiento de la población y el compromiso de la ciudadanía mejora la efectividad y la calidad de las decisiones.

- Desarrollar iniciativas y herramientas innovadoras para que la ciudadanía coopere con las instituciones.

Si bien es cierto que los tres valores son indispensables para un Gobierno abierto, se debe reconocer que la transparencia es el principio básico, y primero, para abrir las instituciones. Si las administraciones pretenden conocer las necesidades de la ciudadanía y permitir que participe y colabore, es un requerimiento indispensable que se establezca una comunicación efectiva con la ciudadanía (Abu-Shamab, 2015). Este es también el motivo por el que el Gobierno abierto está tan ligado con el desarrollo de la tecnología, pues su éxito depende de que la comunicación con la ciudadanía sea efectiva y deben aprovechar las herramientas digitales para tal objetivo.

Aunque Abu-Shamab (2015) señala que existen algunos dilemas que derivan del desarrollo del Gobierno abierto, como la privacidad de la información y la calidad y accesibilidad de los datos, entre otros, cabe destacar las consecuencias beneficiosas. En esta línea, Janssen, Charalabidis,y Zuiderwijk,(2012) subrayan el aprovechamiento de la inteligencia colectiva, pues la deliberación permite la explotación del conocimiento ciudadano y la toma de decisiones legitima las medidas ejecutadas. También progresa la comunicación entre instituciones, a una mayor eficiencia de los servicios y a la estimulación de la competitividad. Todo ello puede incrementar y restaurar la confianza de la ciudadanía en las administraciones y empodera a la población.

Los mismos autores también afirman que la apertura de datos (open data) derivada de los procedimientos del Gobierno abierto conlleva el desarrollo de iniciativas innovadoras, la reutilización de la información, la introducción de nuevos puntos de vista en las administraciones y el crecimiento económico.

En España, donde se centra esta investigación, el Gobierno central promueve diversas iniciativas en el marco de los planes de acción de Gobierno abierto desde que en 2011 pasó a pertenecer al organismo internacional llamado Alianza por el Gobierno abierto (Open Government Partnership). Hasta la fecha, se han efectuado el I Plan de Acción entre 2014 y 2016; el II Plan de Acción entre 2012 y 2014; y el III Plan de Acción, entre 2015 y 2018. 
El IV Plan de Acción de Gobierno abierto de España se desarrollará entre los años 2019 y 2021. Sus principales objetivos son impulsar la participación de la ciudadanía en la toma de decisiones, continuar desarrollando la transparencia y los datos abiertos, fortalecer los valores éticos y de confianza de la ciudadanía y sensibilizar a la sociedad y al funcionariado público de los valores del Gobierno abierto².

\subsection{Partidos políticos: de la transparencia a la apertura}

La publicación de información no solo se ha estudiado y desarrollado en las administraciones públicas, sino que el sector privado también se ha visto influido por la transparencia. Los partidos políticos españoles comenzaron a ofrecer en sus páginas web una sección dedicada a la transparencia en 2011, como señalan Dader, Campos y Quintana (2011).

A ello han contribuido la actividad realizada por diversos organismos que se dedican a promocionar la apertura informativa, como Transparencia Internacional ${ }^{3}$, la Fundación Compromiso y Transparencia ${ }^{4}$ y Dyntra ${ }^{5}$. Estas organizaciones hacen presión a las formaciones a través de la publicación de evaluaciones sobre su transparencia, aunque la falta de un consenso metodológico hace preciso reforzar estas mediciones y valorar no solo la publicidad activa, sino otros aspectos sobre la apertura de las formaciones.

Los partidos políticos deben cumplir con varias obligaciones de transparencia activa desde la aprobación de la Ley 19/2013, de 9 de diciembre, de Transparencia, Acceso a la Información Pública y Buen Gobierno. Pero la normativa resulta insuficiente por las carencias que presenta, como las escasas exigencias de publicidad activa y la ausencia de sanciones ante el incumplimiento de la norma (Cotino, 2014). Es necesario, por tanto, que los partidos políticos se esfuercen en ser más transparentes y abiertos que lo que la propia norma exige y demuestren así ejemplaridad democrática.

Los partidos políticos no deben ser transparentes solo por que estén obligados por la LTABG. La transparencia es un concepto ligado a la eficacia y la honestidad de cualquier actividad política y que, aunque no es la solución, es la base de la restauración de la confianza de la ciudadanía en la clase política.

Se debe destacar que, según el Centro de Investigaciones Sociológicas (CIS), el 45,3\% de la población española considera que los partidos políticos representan uno de los principales problemas en la actualidad. A esto hay que sumar que, en España, los partidos políticos reciben más del $75 \%$ de su financiación de fuentes públicas (Gavilanes, Andrés y Belmonte, 2016). Por tanto, las formaciones deben rendir cuentas a la ciudadanía, que aporta una gran parte de sus ingresos y es, además, a quienes quieren gobernar.

Todos estos motivos refuerzan la idea de que los partidos políticos no solo deben potenciar la transparencia, sino también la participación y la colaboración para permitir el acceso a su información, fomentar el debate, la toma de decisiones y la colaboración con la ciudadanía. Potenciando estas medidas, los partidos lograrán rendir cuentas los ciudadanos, a

\footnotetext{
Gobierno de España (2019). IV Plan de Gobierno abierto.

Fuente: https://transparencia.org.es/evaluacion-partidos-politicos/ (Consulta: 09/03/2020

Fuente: https://www.compromisoytransparencia.com/categorias-informes/partidos-politicos (Consulta: 09/03/2020)

Fuente: https://www.dyntra.org/indices/partidos-politicos/partidos-politicos-elecciones-generales/ (Consulta: 09/03/2020)
}

66 | n³1, pp. 63-86 | doxa.comunicación

julio-diciembre de 2020 
quienes deben informar sobre la finalidad de los recursos que aportan a las formaciones, harán un ejercicio de higiene democrática y fortalecerán la democratización de sus procesos.

\section{Objetivos y metodología}

El objetivo principal de este trabajo consistió en evaluar si los partidos políticos abiertos fomentan los principios del Gobierno abierto. Para ello, en primer lugar, se pretendía elaborar una guía de buenas prácticas con las iniciativas que las formaciones deben seguir para ser considerados abiertos. En segundo lugar, se quería comprobar si los principales partidos políticos del país (Partido Popular, Partido Socialista, Unidas Podemos y Ciudadanos ${ }^{6}$ ) cumplen los mencionados principios. Este trabajo contó, por tanto, con dos partes diferenciadas, para las que se aplicaron diferentes metodologías:

1. Elaboración de la Guía de buenas prácticas para partidos políticos abiertos.

2. Evaluación de los partidos políticos basada en las iniciativas presentes en la Guía.

Para la elaboración de la Guía de buenas prácticas para partidos políticos abiertos, se tuvo en cuenta la ausencia de estudios académicos anteriores respecto a este concepto. Se eligió la metodología Delphi, precisamente porque uno de sus funciones es encontrar respuestas a un problema complejo. El método Delphi consiste en rondas de cuestionarios a personas especializadas en un campo (Astigarraga, 2003).

En primer lugar, se definió el tema de estudio y se seleccionó a un panel de expertos procedentes de diferentes disciplinas (Derecho, Ciencias Políticas, Comunicación) en los campos de la transparencia, la participación ciudadana y la colaboración, a quienes se les planteó su participación en el trabajo. Finalmente, 20 personas respondieron a las dos rondas de cuestionarios. El listado de personas que participaron en el estudio Delhpi está disponible en los anexos de este artículo.

El primer cuestionario tenía como finalidad la definición de los principios de los partidos políticos abiertos, mientras que en el segundo cuestionario se definieron iniciativas concretas y los especialistas evaluaron sus propias respuestas.

Cuando se obtuvieron los resultados de los cuestionarios, se siguió el criterio de Fomin et al. (2008), en el que se combinaron las respuestas, la bibliografía internacional consultada y el criterio propio para elaborar la Guía de buenas prácticas para partidos políticos abiertos. A continuación, se creó una página web (www.partidospoliticosabiertos.com), para fomentar la transparencia del proceso, desde la que se pudieron recibir sugerencias y comentarios para seguir desarrollando la guía.

La segunda parte de esta metodología consistió en evaluar si los partidos políticos mencionados cumplen con lo establecido en la Guía en sus páginas web, para lo cual se realizó una ficha de análisis, cuyos ítems eran cada uno de los principios recomendados anteriormente. Esta ficha de análisis, como estaba basada en la Guía -que se explica en epígrafes posteriores-, contó con tres bloques diferenciados: Bloque I. Transparencia, Bloque II. Deliberación y Bloque III. Toma de decisiones y Colaboración. Salvo en casos excepcionales, los ítems se calificaron con un 2 en caso de cumplimiento, con un 1 en caso de cumplimiento parcial y con un 0 en caso de incumplimiento.

6 Se seleccionaron aquellas formaciones con representación en el Congreso de los Diputados en la XII Legislatura. Solamente se seleccionaron los partidos que se presentaban a nivel nacional. 
A continuación, se explican brevemente los bloques de este análisis:

1. Transparencia. En este apartado se tuvieron en cuenta los elementos publicados por los partidos políticos como publicidad activa, basados en estudios académicos relevantes (Dader et al., 2014; Vaccari, 2011; Larrondo-Urieta, 2016; Pérez et al., 2013), así como en conocidos índices de transparencia (Transparencia Internacional, Fundación Compromiso y Transparencia y Dyntra). La evaluación de la publicidad activa contó con las siguientes categorías:

a. Exigencias de la Ley de Transparencia (14 elementos).

b. Información institucional (22).

c. Procedimientos y organización interna (9).

d. Datos relacionados con la comunicación corporativa (18).

e. Cuentas del partido, obligaciones de la Ley de Financiación de Partidos Políticos e información sobre la contratación (18).

f. Además, también se analizaron otros elementos como la disponibilidad de herramientas de petición de informaciones, el uso de un lenguaje sencillo, la actualización de la información, el uso de formatos reutilizables, así como la accesibilidad de los datos.

2. Deliberación. El segundo bloque estuvo dedicado al fomento del debate entre el electorado, por lo que se tuvo en cuenta si está presente en los estatutos del partido y si existe un código de conducta al respeto. También se valoró la existencia de iniciativas deliberativas, que se explicarán en el próximo epígrafe.

3. Toma de decisiones y Colaboración. En el último bloque se analizó si los conceptos de toma de decisiones y colaboración están presentes en los estatutos del partido y si existen códigos de conducta, al igual que en el anterior caso. Posteriormente, se realizaron listados de iniciativas decisivas y colaborativas y se comprobó si los partidos políticos disponen de estas.

El análisis tuvo una puntuación máxima de 292 puntos, de los cuales el bloque de Transparencia reunió 212 (72,6\% del total): el de Deliberación, 38 (13,01\%); y el de Toma de decisiones y Colaboración, 42 (14,38\%). En este sentido, se consideró un resultado por encima del 50\% es "aceptable", por encima del 65\% es "bueno" y por encima del 85\% es "óptimo".

\section{Resultados}

\subsection{Guía de buenas prácticas para partidos políticos abiertos}

La Guía de buenas prácticas para partidos políticos abiertos muestra las iniciativas que las formaciones deben fomentar su apertura interna y externa. Precisamente con el objetivo de fomentar la transparencia, las recomendaciones están publicadas en una plataforma digital, cuyo enlace es www.partidospoliticosabiertos.com.

Los partidos políticos abiertos son aquellos que son transparentes y publican información actualizada y accesible, rinden cuentas a la ciudadanía, fomentan el debate entre el electorado, permiten a los usuarios participar en su toma de decisiones y realizan actividades colaborativas con los ciudadanos. 
En esta Guía de buenas prácticas cuenta con tres bloques, mencionados anteriormente, que son el de Transparencia, Deliberación y Toma de Decisiones y Colaboración. Los expertos tuvieron que valorar del 1 al 5 la inclusión de las indicaciones en la Guía y, a continuación, se detalla la media obtenida en las recomendaciones.

\section{Figura1: los pilares de los partidos políticos abiertos}

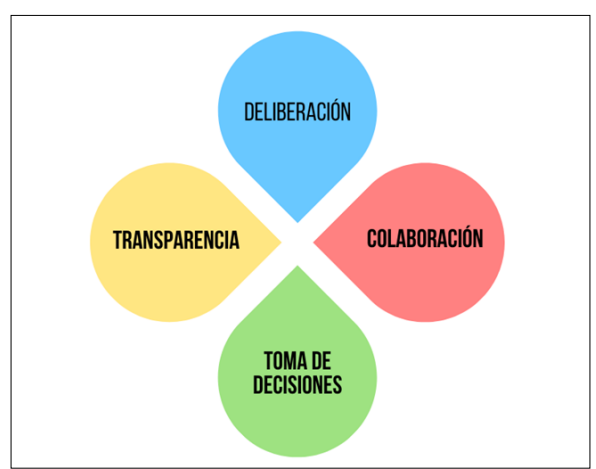

Fuente: elaboración propia a partir de los resultados del estudio Delphi

\section{Bloque I. Transparencia}

El primer bloque está dedicado a la transparencia. Los expertos que participaron en el estudio Delphi valoraron de forma muy elevada (4,69 en una valoración del 1 al 5) la necesidad de que las formaciones sean transparentes. Para mantener a la ciudadanía informada, los partidos políticos deben publicar información en sus portales digitales a través de la publicidad activa. En este sentido, deben publicar la siguiente información:

- Información institucional: datos relativos al partido político, como los estatutos, los valores ideológicos, el código deontológico, la historia del partido, entre otros.

- Procedimientos y organización interna: información sobre el funcionamiento y la organización interna y externa, como el organigrama, el currículum de los miembros de partido, las funciones de los altos cargos, los reglamentos internos, las listas electorales, etc.

- Datos relacionados con la comunicación corporativa: aquellos datos que son especialmente útiles para periodistas y medios de comunicación, aunque también para la ciudadanía interesada, como el calendario de actividades, la agenda de los principales cargos de la formación, una sección de noticias con elementos gráficos y audiovisuales, información parlamentaria, enlaces a las redes sociales, además de otros.

- Cuentas del partido y exigencias de la Ley de Financiación de Partidos Políticos y contratación: deben hacer públicas las exigencias de la Ley de Financiación de Partidos Políticos y los datos de contratación del partido político (instrucciones y contratos realizados). 
En la Ley de Transparencia, Acceso a la Información Pública y Buen Gobierno, se incluye a los partidos políticos como sujetos obligados a cumplir con la publicidad activa, pero no con el acceso a la información. En este sentido, los expertos consideraron $(4,05)$ que las formaciones deberían crear un sistema de peticiones de información reguladas, con plazos de respuesta establecidos, a través de un sistema sencillo y gratuito.

Los portales de los partidos deben contar con un buscador avanzado, que permita buscar información por temática y fecha.

Toda la información que se publica debe estar actualizada, como mínimo de forma semestral, aunque sería interesante que se actualizase mensualmente -con la excepción de las noticias y contenidos similares, que tienen una periodicidad diferente-. Debe utilizarse un lenguaje claro y sencillo, con los mínimos tecnicismo y, si los hay, deben aclararse.

Uno de los aspectos más destacados por los expertos $(4,94)$ fue la necesidad de que el portal sea accesible para todo tipo de personas y que los datos sean útiles y reutilizables. Para ello, se deben difundirse en formatos adecuados y debe evitarse la publicación de archivos PDF.

Uno de los objetivos de los partidos políticos debe ser rendir cuentas a la ciudadanía (4,27). Esto implica que se hagan responsables de sus decisiones, cumplan sus promesas y sometan sus actividades al escrutinio público. Un ejemplo de rendición de cuentas que los expertos apoyaron (4) consiste en publicar el cumplimiento del programa electoral.

\section{Bloque II. Deliberación}

El segundo bloque de la Guía se refiere a la promoción de la deliberación. Para los expertos que participaron en el estudio Delphi, la deliberación supone que los partidos fomenten el debate entre el electorado. Se requiere de ellos una gran implicación, de forma que la deliberación sea libre, plural y basada en procesos transparentes $(4,83)$. Esta implicación se debe traducir en la puesta en marcha de recursos para el debate, su inclusión en los estatutos del partido (3,55), así como en la creación de un código de conducta o un reglamento que regule los procesos $(4,44)$.

La deliberación, según lo expuesto por los expertos, conlleva una relación de cercanía entre la clase política y la ciudadanía, el planteamiento de nuevas ideas, la renovación de agendas y el aumento de la eficacia. Cabe señalar que la deliberación no solamente debe ocurrir en las plataformas digitales, sino también presencialmente $(4,88)$, aunque todos los procesos deberían publicitarse para aumentar su promoción, su eficacia y garantizar la transparencia.

Los partidos políticos pueden promocionar la deliberación con diversas iniciativas, a saber: 
Tabla 1: iniciativas deliberativas para partidos políticos abiertos

\begin{tabular}{|c|c|}
\hline Iniciativas deliberativas & $\begin{array}{l}\text { Valoración media de los } \\
\text { expertos (del } 1 \text { al 5) }\end{array}$ \\
\hline Chats debates y preguntas a miembros del partido. & 4,06 \\
\hline $\begin{array}{l}\text { Plataforma de propuestas. En ellas los usuarios pueden enviar ideas que consideran necesarias a } \\
\text { otros simpatizantes y pueden debatir sobre estas. Para ello, se requiere una figura de moderador que } \\
\text { garantice la pluralidad y el respeto. }\end{array}$ & 4,33 \\
\hline Foros de debate. & 4,13 \\
\hline Secciones de comentarios. & 3,4 \\
\hline Opción de compartir contenido. & 3 \\
\hline \multicolumn{2}{|l|}{ Alertas } \\
\hline Telegram & 3,07 \\
\hline WhatsApp & 2,7 \\
\hline Correo electrónico & 2,6 \\
\hline Contacto por e-mail. & 3,06 \\
\hline Aplicaciones como Reddit & 3,8 \\
\hline $\begin{array}{l}\text { Eventos offline. } \\
\text { - } \quad \text { Reuniones periódicas de líderes con simpatizantes. } \\
\text { - } \text { Mesas redondas. } \\
\text { - Grupos debate. } \\
\text { - } \text { Jornadas informajo. } \\
\text { Congresos. }\end{array}$ & 4,46 \\
\hline
\end{tabular}

Fuente: elaboración propia a partir de los resultados del estudio Delphi

\section{Bloque III. Toma de decisiones y Colaboración}

El último bloque está compuesto por dos conceptos diferenciados. Según lo expuesto por los expertos, la toma de decisiones se entiende desde el punto de vista clásico de la participación, en que los ciudadanos pueden elegir propuestas. En cambio, en la colaboración, pueden tomar parte de las actividades, es decir, crear conjuntamente con los partidos políticos. Para ambos conceptos se requiere la transparencia, así como la existencia de procesos deliberativos.

\section{Toma de decisiones}

La toma de decisiones permite a la ciudadanía participar activamente en las medidas de los partidos políticos. Son necesarios procesos regulados, así como la identificación de los usuarios y su protección. Además, no solo se debe dar a través de Internet, sino también de forma presencial $(3,88)$.

Un partido abierto debe contar con procedimientos de democracia interna. También se considera que las siguientes iniciativas permiten la toma de decisiones: 
Tabla 2: iniciativas de toma de decisiones para partidos políticos abiertos

\begin{tabular}{|l|c|}
\hline Iniciativas de toma de decisiones & $\begin{array}{c}\text { Valoración media de los } \\
\text { expertos (1-5) }\end{array}$ \\
\hline $\begin{array}{l}\text { Consultas ciudadanas: esta es una forma clásica de participación, en la que la } \\
\text { ciudadanía puede votar de forma afirmativa o negativa la propuesta del partido, } \\
\text { elegir entre diversas opciones (como una lista de candidatos), etc. Se debe } \\
\text { facilitar su disponibilidad también de forma presencial, como se ha mencionado } \\
\text { anteriormente. }\end{array}$ & 4,6 \\
\hline Recogidas de firmas a través de sistemas adecuados. & 3,2 \\
\hline $\begin{array}{l}\text { Aplicaciones digitales que permiten participar activamente en la toma de } \\
\text { decisiones. }\end{array}$ & 3,8 \\
\hline
\end{tabular}

\section{Fuente: elaboración propia a partir de los resultados del estudio Delphi}

\section{Colaboración}

Los expertos señalaron en el estudio Delphi que los partidos pueden promover iniciativas para que la ciudadanía les ayude en la ejecución y la ejecución de diferentes actividades. De nuevo, estas pueden ser digitales o no $(4,38)$. La colaboración permite que los partidos se beneficien de los conocimientos de los usuarios y simpatizantes, establecen lazos con ellos y les implican en las actividades de la formación.

\section{Tabla 3: iniciativas colaborativas para partidos políticos abiertos}

\begin{tabular}{|l|c|}
\hline Iniciativas de colaboración & $\begin{array}{c}\text { Valoración media de los } \\
\text { expertos (1-5) }\end{array}$ \\
\hline Colaboración en la elaboración de programas electorales. & 4,71 \\
\hline Buzón de quejas y sugerencias. & 3,33 \\
\hline Encuestas. & 3,7 \\
\hline Envío de materiales. & 2,53 \\
\hline Recaudación de fondos online / Donativos. & 3,85 \\
\hline Crowdsourcing o herramientas para la distribución de tareas. & 4,3 \\
\hline Enlace a aplicaciones o herramientas que hayan reutilizado datos del partido. & 3,76 \\
\hline Colaboración en campañas electorales. & 4,14 \\
\hline Eventos offline: laboratorios de innovación y talleres de trabajo, entre otros. & 4,71 \\
\hline Herramientas wiki. & 3,84 \\
\hline Acción social. & 4,16 \\
\hline Redes sociales a través de campañas de colaboración. & 3,64 \\
\hline Campañas de movilización. & 3,85 \\
\hline
\end{tabular}

Fuente: elaboración propia a partir de los resultados del estudio Delphi 


\subsection{Evaluación de la apertura de los partidos políticos}

Redactada la Guía de buenas prácticas para partidos políticos abiertos, se procedió a analizar las páginas web de los cuatro partidos seleccionados: Partido Popular, Partido Socialista, Unidas Podemos y Ciudadanos. Este análisis se realizó entre los meses de abril y mayo de 2019, por lo que coincidió con las Elecciones Municipales, Autonómicas, Europeas, así como con las Generales, que fueron adelantadas -se debían haber celebrado en junio de 2020-. Esto pudo ser un factor favorecedor para los partidos políticos, que en campaña electoral realizan más actividades relacionadas con la comunicación que acercan a las formaciones al electorado.

Solamente un partido suspendió la evaluación total de la apertura de los partidos políticos. Ciudadanos obtuvo un 49,37\% (144,15 puntos), con tan solo el primer bloque (Transparencia) aprobado y solo de una forma aceptable, con un $57,08 \%$ $(122,15)$. En cuento a la Deliberación y la Toma de decisiones y la Colaboración, ambos niveles fueron muy deficientes, con un $26,31 \%$ (10) y un 28,57\% (12), respectivamente.

Algo mejor que Ciudadanos y con el cómputo global aprobado, el Partido Popular obtuvo un 54,35\% de los elementos analizados (158,7). Solamente suspendió el tercer bloque (Toma de decisiones y Colaboración), con un escaso 16,6\% (7), mientras que en Transparencia alcanzó un 62,59\% (132,7) y en Deliberación, un ajustado 50\% (19). Aun así, el $P P$ no alcanzó en ningún nivel un resultado positivo u óptimo, según lo valorado en la metodología.

El PSOE, por su parte, tuvo un resultado total de 63,53\% (185,52). El bloque de transparencia fue positivo, con un 69,58\% (148,52), seguido de un nivel aceptable en Deliberación, con un 57,89\% (22). Sin embargo, el bloque de Toma de decisiones y Colaboración fue insuficiente, con un 25,71\% (15).

Unidas Podemos fue el único partido que aprobó todos los bloques y también el que mejor resultado tuvo globalmente, con un $66,74 \%(194,89)$. Así, los niveles de Transparencia y de Deliberación fueron positivos, con 69,28\% (146,89) y 65,78\% (25), respectivamente. En Toma de decisiones y Colaboración solo tuvieron un 54,76\% (23). Cabe destacar, no obstante, el esfuerzo de esta formación, pues fue la única con un resultado aceptable en el último bloque.

Tabla 4: resultado final en el análisis de la apertura de los partidos políticos

\begin{tabular}{|c|c|c|c|c|c|c|c|c|}
\hline & \multicolumn{2}{|c|}{ Partido Popular } & \multicolumn{2}{|c|}{ Partido Socialista } & \multicolumn{2}{c|}{ Unidas Podemos } & \multicolumn{2}{c|}{ Ciudadanos } \\
\hline Transparencia (212) & 132,7 & $62,59 \%$ & 148,52 & $69,58 \%$ & 146,89 & $69,28 \%$ & 122,15 & $57,08 \%$ \\
\hline Deliberación (38) & 19 & $50,00 \%$ & 22 & $57,89 \%$ & 25 & $65,78 \%$ & 10 & $26,31 \%$ \\
\hline $\begin{array}{c}\text { Toma de decisiones y } \\
\text { Colaboración (42) }\end{array}$ & 7 & $16,60 \%$ & 15 & $35,71 \%$ & 23 & $54,76 \%$ & 12 & $28,57 \%$ \\
\hline Total (292) & 158,7 & $54,35 \%$ & 185,52 & $63,53 \%$ & 194,89 & $66,74 \%$ & 144,15 & $49,37 \%$ \\
\hline
\end{tabular}

Fuente: elaboración propia 
A continuación, se explican los resultados de los niveles de apertura de los partidos políticos:

\section{Bloque I. Transparencia}

El bloque relativo a la transparencia es el que más peso tuvo en este análisis de las plataformas digitales de los partidos políticos. También fue en el que mejores resultados obtuvieron todas las formaciones y todos ellos tuvieron puntuaciones satisfactorias, si bien las del PSOE y Unidas Podemos estuvieron por encima. El PSOE logró el 69,58\% (148,52 puntos sobre 212), prácticamente empatado por Unidas Podemos, con 69,28\% $(146,89)$. Les seguían el PP con el 62,59\% $(132,7)$, y Ciudadanos, con 57,08\% (122,15). Todos los partidos estuvieron por encima del 50\% y PSOE y Unidas Podemos, por encima del $65 \%$, por lo que se considera que sus resultados fueron positivos. Así, se observa un esfuerzo por parte de las formaciones en el desarrollo de este pilar.

Gráficol: resultados del Bloque I (Transparencia)

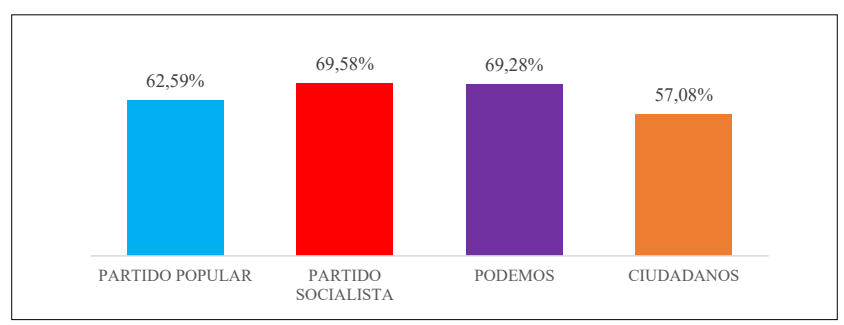

Fuente: elaboración propia

Para analizar la transparencia, se comprobó la publicación de los diferentes tipos de información que las formaciones deben difundir, anteriormente mencionados. En este sentido, la publicación de las exigencias de la Ley de Transparencia, Acceso a la Información Pública y Buen Gobierno tuvo una media positiva, de 69,64\%, aunque fue muy irregular, ya que Ciudadanos y el $P P$ solo publicaron el 53,57\% de los elementos solicitados. En cambio, Unidas Podemos difundió prácticamente todos (92,85\%) y el $P S O E$, el $78,57 \%$.

Las principales carencias consistieron en la ausencia de los contratos con la Administración Pública y los convenios suscritos con esta, así como los programas anuales y su grado de cumplimiento, un elemento que ayudaría a rendir cuentas a la ciudadanía.

El apartado de información institucional recibió un resultado más unánime y más positivo, con una media del 79,54\%, y puntuaciones muy similares por parte de todas las formaciones: PP, 86,3\%; PSOE, 77,27\%; Unidas Podemos, 79,54\%; y Cs, $75 \%$. Se valoró especialmente la tarea de las formaciones en esta línea, aunque llama la atención la ausencia de algunos elementos, como la publicación de la historia del partido por parte de Unidas Podemos. Cabe destacar de forma 
positiva la forma en la que el $P P$ publica su posicionamiento ante ciertos temas o medidas, pues disponen de una sección propia llamada 'Temas', en donde aparecen agrupadas las publicaciones, documentos y aclaraciones respecto a diversas temáticas.

Figura 2: posicionamiento ante políticas de igualdad del Partido Popular

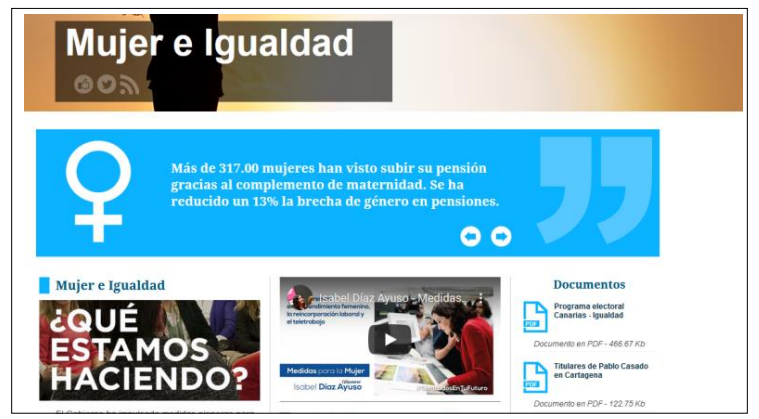

Fuente: http://www.pp.es

La publicación de información de procedimientos y organización interna descendió y tuvo una media de 56,94\%, con resultados irregulares por parte de las formaciones. Unidas Podemos publicó el 66,66\% de los elementos; el PSOE, el 51,11\%; Ciudadanos, el 55,55\%; y el PP suspendió, con un 44,44\%. Un detalle que resultó llamativo es que Unidas Podemos no publicase un calendario de eventos del partido y sí publicase la agenda del presidente, Pablo Iglesias. También lo Ciudadanos lo hizo con Albert Rivera, aunque ambos partidos tuvieron una actualización algo deficiente, mientras que el PSOE y el $P P$ no lo publicaron las agendas de sus respectivos dirigentes.

También se valoró la publicación de información relacionada con la comunicación corporativa, cuya media fue de un 59,72\%. El partido con mejores resultados fue el PSOE, con un 77,77\%, seguido de Unidas Podemos (61,11\%), el PP (52,77\%) y Ciudadanos (47,22\%).

Destaca, en este sentido, la sección de actualidad del PSOE que, aun con algunas carencias, contaba con un interesante apartado de noticias, un archivo de publicaciones pasadas, una galería fotográfica y audiovisual, streaming de eventos y archivos de audio. Todo ello no solamente acerca a la formación a la ciudadanía interesada, sino que también es especialmente útil para los medios de comunicación y los periodistas en general, por las facilidades que implica en la realización de su trabajo.

El último apartado de publicidad activa fue el relativo a las cuentas del partido, en el que todas las formaciones obtuvieron el mejor resultado del análisis, salvo Unidas Podemos. La media fue del $85,41 \%$, con el $P P$ a la cabeza $(94,44 \%)$, seguido del PSOE (88,88\%), Ciudadanos (83,33\%) y Unidas Podemos (75\%). La principal escasez correspondió a la información relativa a la contratación, ya que todos los partidos, salvo Unidas Podemos, publicaron las instrucciones internas sobre contratación, pero ninguna informó sobre los contratos realizados y solo el $P S O E$ y el $P P$ difundieron las bases de contratación. 
Tabla 5: resultados de publicidad activa

\begin{tabular}{|c|c|c|c|c|}
\hline & $\begin{array}{l}\text { Partido } \\
\text { Popular }\end{array}$ & Partido Socialista & Unidas Podemos & Ciudadanos \\
\hline Exigencias de la Ley de Transparencia & $53,57 \%$ & $78,57 \%$ & $92,85 \%$ & $53,57 \%$ \\
\hline Información institucional & $86,36 \%$ & $77,27 \%$ & $79,54 \%$ & $75 \%$ \\
\hline Procedimientos y organización interna & $44,44 \%$ & $61,11 \%$ & $66,66 \%$ & $55,55 \%$ \\
\hline Datos relacionados con la comunicación corporativa & $52,77 \%$ & $77,77 \%$ & $61,11 \%$ & $47,22 \%$ \\
\hline
\end{tabular}

Fuente: elaboración propia

Se hallaron más deficiencias en el acceso a la información que en la publicidad activa, pues ningún partido contó con un sistema de petición de información, que contara con plazos de respuesta y un sistema regulado. La única opción para ello eran las diversas cuentas de correo electrónico a las que los usuarios pueden escribir en caso de querer alguna información. Sin embargo, al no existir un sistema establecido, con normas y periodos de respuesta, los ciudadanos pueden no recibir respuesta o recibirla muy tarde.

Se consideró la calidad de los buscadores en estas páginas web y el más avanzado fue el del Partido Popular, pues permitía buscar la información por temática y por fecha. Llamó la atención que Ciudadanos ni siquiera tuviera buscador, algo que se considera esencial.

En cuanto a la actualización, también es un elemento que deben mejorar los partidos políticos, pues solo Unidas Podemos incluyó la fecha en la que se había actualizado la información en algunos de los contenidos y, aun así, eran fechas algo lejanas (incluso un año antes de la fecha en la que se efectuó el análisis). Es necesario que los partidos actualicen más sus contenidos y lo indiquen en sus portales.

El uso de un lenguaje sencillo y sin tecnicismos fue algo que todas las formaciones respetaron, aunque ninguna contó con una herramienta específica para aclarar términos concretos (por ejemplo, cuadros de texto emergentes).

La reutilización de la información fue uno de los puntos débiles de los partidos políticos, entre los que destacó Unidas Podemos con el uso del software Tableau para la visualización de los datos de una forma más dinámica. Aun así, este solo estaba disponible en el Portal de Transparencia y no en el resto de la página web.

En cuanto a la accesibilidad, la publicación de la web en otros idiomas de España solo se llevó a cabo por Ciudadanos y Unidas Podemos y este último fue el único que también la publicó en inglés.

Se midió también si los partidos políticos muestran su compromiso con la transparencia publicando resultados de rankings o acuerdos relativos a este tema, algo que solo realizaron el PSOE y Ciudadanos. Además, Unidas Podemos mencionó el cumplimiento de la Ley de Transparencia en su sección 'Cuentas claras'. El PSOE, por su parte, contó con una pequeña sección en su web en la que defiende las mejoras que requiere la norma. 
La rendición de cuentas es una de las cuentas pendientes para los partidos políticos, pues ninguno de ellos publicó el grado de cumplimiento de su programa electoral, ni de otros planes políticos. Esto podría ser muy relevante, pues de esta forma serían claros en cuanto al ejecución de sus promesas y, al publicarse, podría provocar una mayor eficacia y cumplimiento de las mismas.

\section{Bloque II. Deliberación}

El segundo nivel de este análisis es el relativo al debate entre el electorado y los partidos políticos. En este bloque se pudieron observar muchas diferencias entre unos partidos y otros. El resultado medio fue del 50\%, es decir, una puntuación justa para aprobarlo. Y es que Unidas Podemos duplicó el resultado de Ciudadanos, con un 65,78\% de los elementos cumplidos (25 puntos), respecto al $26,31 \%$ de $C s$ (10). El $P S O E$ obtuvo un 57,89\% (22) y el $P P$, un $50 \%$ (19).

Gráfico 2: resultados del Bloque II (Deliberación)

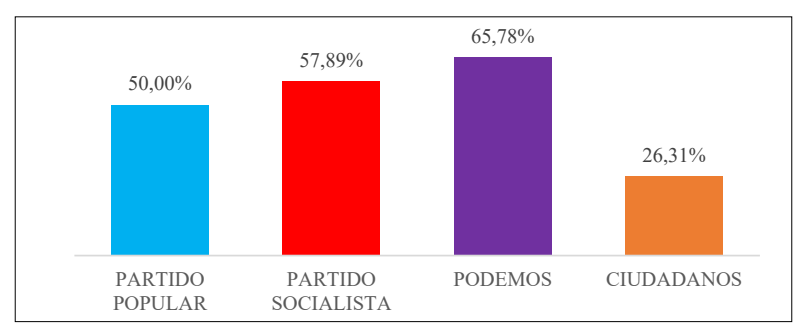

Fuente: elaboración propia

La presencia de la deliberación en los estatutos de los partidos fue irregular. Así, en el de Ciudadanos no hubo ninguna mención, mientras que en el $P P$ solo se señaló la importancia de que los afiliados muestren sus opiniones y participen. El PSOE también mencionó la deliberación tanto como derecho como deber de los afiliados. Unidas Podemos lo definió como uno de los principios generales de la formación.

Ninguno tuvo reglamentos específicos para las iniciativas deliberativas, si bien tanto el PSOE como Unidas Podemos sí disponían de reglamentos internos en los que se menciona alguna de estas medidas, aunque de una forma muy sutil.

Para analizar la presencia de iniciativas deliberativas, así como para las decisivas y colaborativas (tercer nivel), fue necesario registrarse en las plataformas de participación de los partidos políticos. De esta forma se pudieron observar las herramientas dentro de MiPSOE, el espacio socialista, la Plaza Podemos 2.0 y el Espacio Naranja de Ciudadanos. El Partido Popular no disponía de una plataforma similar.

A continuación, se explicará la disposición de las iniciativas deliberativas consideradas en la metodología del trabajo:

Ni el PP ni el PSOE contaron con chats o plataformas de preguntas para los miembros del partido. Ciudadanos sí lo había realizado hace años en eventos del partido, aunque Unidas Podemos fue el que disponía de una plataforma más interesante. Llamada Escaño Abierto: tu voz en el parlamento, esta herramienta cede a la ciudadanía algunas de las preguntas que Unidas Podemos realiza en la Asamblea de Madrid, de forma que esas cuestiones se responden por parte del parlamento madrileño. Aunque esta no es exactamente una plataforma de preguntas y respuestas y solo afecta a Madrid, se 
incluyó en este apartado dada su innovación. Sería innovador que las formaciones siguieran este camino y se interesaran por las preocupaciones e ideas de la ciudadanía.

Unidas Podemos fue el único partido con herramientas para realizar propuestas. En concreto, tenían dos, una llamada Iniciativas Ciudadanas y otra, Iniciativas Ciudadanas Populares. La primera fue sustituida por la segunda, aunque el archivo sigue en la plataforma de participación. El funcionamiento es el mismo: los inscritos pueden hacer propuestas y, si tienen apoyos suficientes, pueden pasar a debatirse en un referéndum vinculante. Además, las personas inscritas pueden comentar las iniciativas propuestas por otros.

Todas las formaciones, salvo Ciudadanos, pusieron a disposición de los usuarios la posibilidad de compartir el contenido en diversas redes sociales en la mayor parte de sus seccione -Cs solo lo hizo en la de Actualidad- y ninguno permitió comentar las secciones.

Esta investigación también indagó en posibles innovaciones tecnológicas que los partidos políticos puedan aplicar a la deliberación. Por ello, se comprobó la existencia de chatbots o asistentes virtuales (Campos-Domínguez y García-Orosa, 2018), pero ninguna formación contó con ello. Esto supondría un gran avance tecnológico y un paso más allá en la relación de la transparencia y la robótica.

Las herramientas con las que sí que contaron todos los partidos fueron los servicios de alertas a través de Telegram y WhatsApp, por lo que se puede confirmar que está extendido entre las formaciones españolas. Aun así, se debe destacar que en el caso de $C s$, su canal de WhatsApp no aparecía mencionado en su página web, sino en una publicación en Facebook del partido. Asimismo, todos, salvo Ciudadanos, contaron con un servicio de alertas a través del correo electrónico o un sistema de RSS. La formación liderada por Albert Rivera solo ofreció el envío de información del partido a las personas que se hicieran simpatizantes.

Tal y como se esperaba, las cuatro formaciones pusieron a disposición de la ciudadanía el contacto a través del correo electrónico o un formulario de contacto equiparable.

Respecto a la existencia de plataformas digitales de debate, solamente Unidas Podemos contó con una en Reddit (https:// www.reddit.com/r/podemos/), en donde los usuarios pueden publicar mensajes sobre diferentes temáticas, comentar otras publicaciones y votar a favor o en contra de estas.

Se tuvieron en cuenta también los eventos presenciales de deliberación, ya que el debate no solo existe en Internet. Se pudo comprobar a través de las páginas web que el $P P$ y el $P S O E$ realizaron reuniones periódicas con simpatizantes y distintos colectivos, así como diferentes jornadas informativas. Los socialistas, además, también realizaron foros de debate presenciales. Todos los partidos, salvo Ciudadanos, habían celebrado congresos antes de realizar el análisis y disponían de información relacionada en la web. Además, al realizarse este análisis durante la campaña electoral, se tuvo en cuenta también la celebración de los mítines.

Estos resultados muestran que todos los partidos deben seguir potenciando la deliberación, especialmente el Partido Populary, sobre todo, Ciudadanos, pues no obtuvieron resultados suficientemente satisfactorios. No puede olvidarse que 
la deliberación es la base de cualquier debate participativo y, por ello, se deben desarrollar iniciativas que promuevan el debate con la ciudadanía.

\section{Bloque III. Toma de decisiones y Colaboración}

El último nivel, que estudiaba las medidas participativas y colaborativas de las formaciones políticas, fue el peor para todos los partidos. La media estuvo suspensa, con un 33,92\%, Solo Unidas Podemos alcanzó un nivel aceptable, con un 53,76\% (15 puntos), es decir, un resultado muy ajustado. El PSOE obtuvo el 35,71\% de los elementos posibles (15); Ciudadanos, el 28, 57\% (12); y el PP, el 16,6\% (7). Unidas Podemos, con una puntuación que no era muy alta, llegó a triplicar el resultado del Partido Popular, algo que demuestra la falta de compromiso de las formaciones en esta línea.

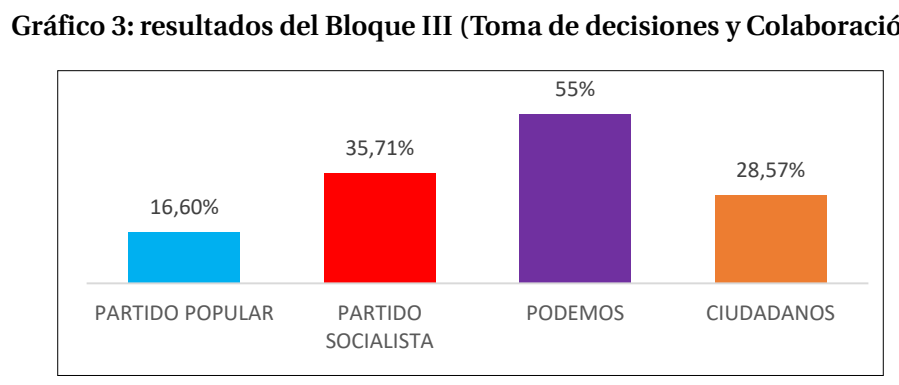

Fuente: elaboración propia

Como en el anterior bloque, también se revisó la presencia de la toma de decisiones y la colaboración en los estatutos de los partidos políticos. El PSOE, Unidas Podemos y Ciudadanos sí que lo mencionaron. Estas tres formaciones disponen de procesos de democracia interna y, por tanto, lo mencionan en los estatutos. En cuanto a los sistemas colaborativos, se mencionó la posibilidad de participar en campañas electorales, así como en la recaudación de fondos y en otras actividades. Mientras tanto, el $P P$ sí mencionó el proceso de democracia interna con el proceso democrático creado para elegir el candidato a la Presidencia del Gobierno - un proceso establecido solo en una ocasión-, así como los de los afiliados de colaborar en las campañas electorales y la posibilidad de que diferentes expertos aporten su conocimiento.

Todos los sistemas tienen estos sistemas de democracia interna regulados, pero ninguno contó con una regulación de los procesos colaborativos de forma específica.

Como se ha mencionado anteriormente, se valoró como una parte esencial de la toma de decisiones la existencia de procesos regulados de democracia interna del partido, algo con lo que contaron el PSOE, Unidas Podemos y Cs, tanto para la Presidencia del Gobierno, como para procedimientos locales y autonómicos. En cambio, en el $P P$ solamente contaron con este sistema para el candidato a la Presidencia del Gobierno, establecido en el $18^{\circ}$ Congreso Popular y celebrado en febrero de 2017.

Respecto a las iniciativas de toma de decisiones investigadas, ni el PP ni Ciudadanos realizaban consultas ciudadanas, mientras que el PSOE había realizado recientemente una, pero se trató de una consulta eventual. Unidas Podemos, en cambio, sí dispone de un sistema de consulta a sus inscritos, que utilizan con cierta frecuencia, en su portal de participación. 
Figura 3: resultado de una consulta de Unidas Podemos

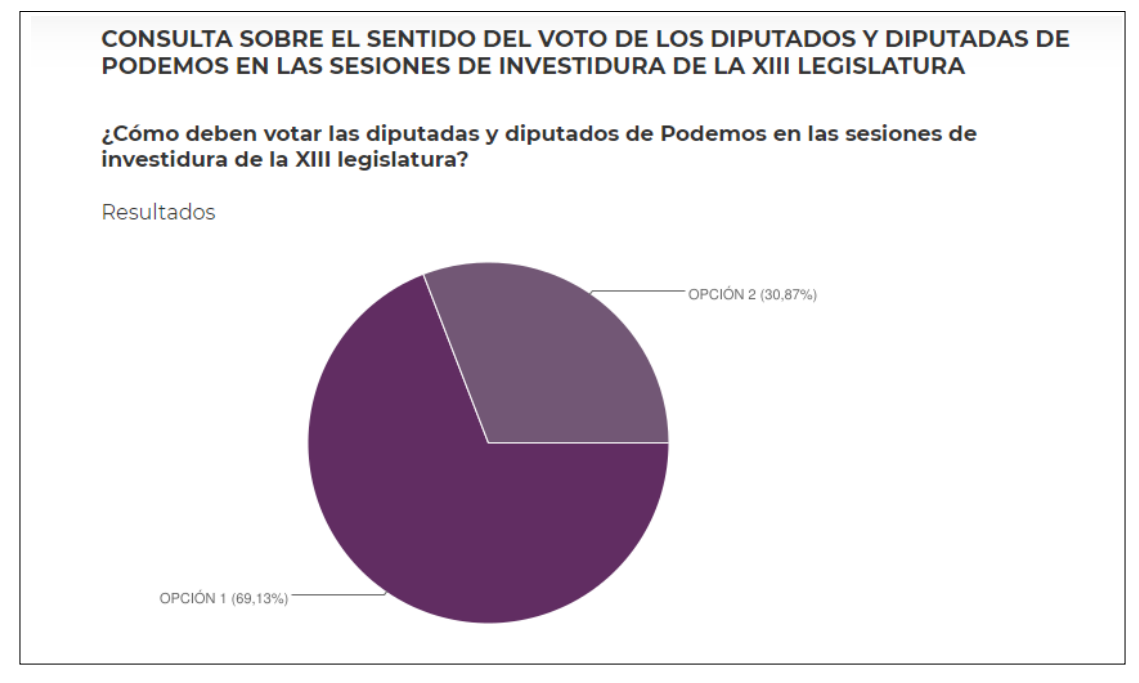

Fuente: https://podemos.info/consulta-estatal-voto-podemos-xiii-legislatura/ (Consulta: 09/03/2020)

Por otro lado, no se halló ninguna recogida de firmas por parte de las formaciones políticas para proponer Iniciativas Legislativas Populares.

En cuanto a aplicaciones digitales, Unidas Podemos fue el único que utilizó NVotes para algunas consultas y un software libre llamado Consul.

Tras analizar las iniciativas de toma de decisiones, se tuvieron en cuenta las relacionadas con la colaboración:

En este sentido, ninguna formación ofreció a la ciudadanía o los simpatizantes una herramienta para proponer medidas del programa electoral, aunque Unidas Podemos sí tenía un sistema para que los inscritos votaran favorable o negativamente al programa propuesto por el partido.

Solo el PSOE contó con un sistema para presentar quejas o sugerencias en su plataforma de participación, mientras que el resto de formaciones solo permitían hacerlo a través del correo electrónico. 
Figura 4: herramienta de quejas y sugerencias del PSOE

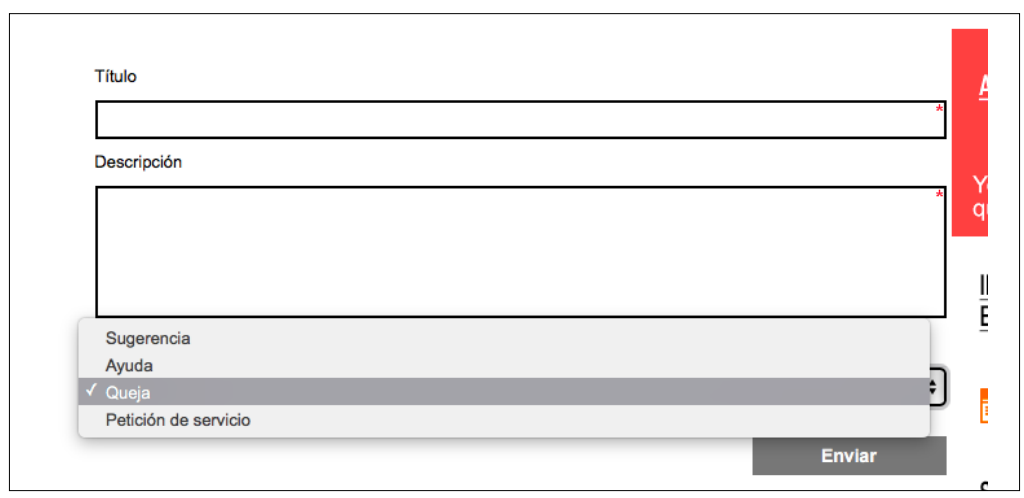

Fuente: https://mipsoe.es/SitePages/Sugerencias.aspx (Consulta: 09/03/2020)

Solamente se halló en el caso del PSOE un espacio dedicado a hacer encuestas, si bien este se había usado por última vez en 2014.

Ningún partido contó con medidas para el envío de materiales, herramientas wiki o de crowdsourcing, que permiten beneficiarse de la voluntad y el conocimiento de los ciudadanos.

Unidas Podemos fue el único partido que publicitó en su web la colaboración en campañas electorales presenciales. Por su parte, $P S O E$, Unidas Podemos y Cs tuvieron redes organizadas para promocionar mensajes en redes sociales. El $P P$ solamente difundió las etiquetas usadas en las redes.

Unidas Podemos fue, de nuevo, la única formación que ofreció un sistema para colaborar en campañas de acción social. Se trata del programa IMPULSA, que financia proyectos sociales innovadores con el excedente de los cargos públicos del partido. Esta iniciativa permite presentarse a organizaciones sin ánimo de lucro y a los Círculos y Consejos Ciudadanos de la formación que desarrollen actividades sociales?.

Por último, ninguna formación promovió iniciativas colaborativas presenciales, como talleres, laboratorios y eventos similares.

\section{Conclusiones y discusión}

Este trabajo supone un primer acercamiento al concepto de partidos políticos abiertos, que hasta ahora no había estado presente en ningún trabajo académico. Por ello, se pretende seguir evaluando la apertura de las formaciones políticas periódicamente, para que exista un registro de su grado de desarrollo a lo largo de los años.

7 Fuente: https://impulsa.podemos.info/wp-content/uploads/2017/06/Bases_Impulsa_Versión_170629.pdf 
Tras la realización del estudio Delphi para la creación de la Guía de buenas prácticas para partidos políticos abiertos se llegó a una definición de cuáles deben ser las características cumplidas por las formaciones para ser consideradas abiertas. Según los expertos, un partido político abierto es aquel que demuestra una actitud de apertura de sus procesos externos e internos de cara a la ciudadanía. Para ello, son transparentes respecto a su información y rinden cuentas a la ciudadanía. También fomentan la deliberación, de forma que prestan información suficiente a la población par que la discusión establecida esté basada en el conocimiento de los asuntos que se debaten. Los partidos abiertos otorgan a la ciudadanía el poder de tomar algunas de las decisiones de las formaciones y promueven la colaboración con colectivos de distinto tipo. Esta apertura conlleva una mayor democratización de los procesos de los partidos políticos y un acercamiento a la ciudadanía.

En este caso, aunque el resultado de la apertura total de las formaciones esté por encima del $50 \%$, con un $58,49 \%$-salvo en el caso de Ciudadanos- presentan importantes deficiencias en el bloque de Deliberación y en el de Toma de decisiones y Colaboración, donde obtuvieron de media un ajustado $50 \%$ y un 33,92\%, consecutivamente.

Unidas Podemos fue el único partido que aprobó los tres bloques, con una media final de $66,74 \%$, seguido del PSOE, con 63,53\%, el Partido Popular, con 54,35\%, y Ciudadanos, con 49,37\%. El esfuerzo que los partidos han realizado en mejorar la transparencia en los últimos años -y que debe seguir desarrollándose- debe hacerse palpable también en sus procesos participativos y colaborativos. Así, resulta necesario que esta apertura no solo consista en el cumplimiento formal de los indicadores propuestos en esta investigación, sino en el compromiso con la ciudadanía de acercarse a esta y rendirles cuentas a través de los tres pilares.

Respecto a la transparencia, que fue el bloque más desarrollado por todas las formaciones, llama la atención que el apartado de publicidad activa relativo a las exigencias de la Ley de Transparencia no fuera el que mejores resultados obtuvo, sino que fue el de las cuentas del partido, con un $85,41 \%$. Esto puede deberse a que en este último análisis se incluyeron las exigencias de la Ley de Financiación de los partidos políticos. Esta normativa, a diferencia de la LTABG cuenta con procesos de sanción, motivo que explica que esta no estuviera tan desarrollada. Por ello, se hace evidente la necesidad de incluir sanciones en caso de incumplimiento en la LTABG.

Tabla 6: resultados totales en los apartados de publicidad activa

\begin{tabular}{|l|c|}
\hline Publicidad activa & Total \\
\hline Exigencias de la Ley de Transparencia & $69,64 \%$ \\
\hline Información institucional & $79,54 \%$ \\
\hline Procedimientos y organización interna & $56,94 \%$ \\
\hline Datos relacionados con la comunicación corporativa & $59,72 \%$ \\
\hline Cuentas del partido & $85,41 \%$ \\
\hline
\end{tabular}

Fuente: elaboración propia 
Sin duda, hay cuestiones que deben mejorar. Destaca la ausencia de procedimientos para realizar peticiones de informaciones a los partidos políticos. Sería muy interesante que la normativa los incluyera también en el capítulo de Acceso a la Información para fomentarlo. También la accesibilidad de los datos publicados por los partidos requiere mejoras, pues se debe publicar la información en formatos reutilizables (y evitar los PDF) y contar con mejores buscadores, sobre todo en el caso de Unidas Podemos, cuya herramienta es muy sencilla, y de Ciudadanos, que no tiene.

Una de las ideas que plantea esta investigación -y que ningún partido tiene- es la publicación del grado de cumplimiento y ejecución de los programas electorales, que garantizaría la rendición de cuentas de los mismos e impulsaría que los políticos se hicieran responsables de sus promesas. Se trata de una iniciativa necesaria para que los programas electorales sean más realistas y que los partidos políticos se centren más en las propuestas que realizan.

Aunque los bloques de Deliberación y Toma de decisiones y Colaboración presentaron deficiencias, cabe destacar algunas iniciativas interesantes llevadas a cabo por las formaciones:

- Plataforma de propuesta de Unidas Podemos, en la que los usuarios pueden presentar iniciativas y debatirlas con otros usuarios.

- Consultas ciudadanas para ciertas decisiones. Unidas Podemos la utiliza más, aunque el PSOE también ha realizado alguna en el pasado.

- Redes de simpatizantes para promocionar al partido en redes sociales, algo que tienen tanto el PSOE como Unidas Podemos y Ciudadanos.

- Plataforma para enviar quejas y sugerencias del $P S O E$, que permite conocer fallos y mejoras al partido.

- Iniciativa para apoyar causas sociales de Unidas Podemos, llamada Impulsa, que fomenta este tipo de acciones.

Los partidos políticos aún deben seguir desarrollando su apertura y deben incentivar. No solo deben incentivar la transparencia como corresponde al amparo de la Ley 19/2013 -que ni siquiera se cumple al completo-, sino que resulta necesario reforzar las iniciativas que tienen que ver con la interacción de la población en sus procesos. Ello les acercará a la ciudadanía y les permitirá beneficiarse del conocimiento colectivo. La apertura de los partidos políticos debe hacerse de la mano de la tecnología y, por tanto, se debe fomentar la innovación digital para tal fin.

Se abre un camino en el plano académico en el estudio de los partidos políticos y su apertura, que hasta ahora se ha centrado más en su publicidad activa. Surgen nuevos aspectos que abordar, especialmente en la colaboración con la ciudadanía, que es el pilar menos desarrollado por las formaciones. Sería muy interesante investigar procesos como la cooperación en el desarrollo de programas electorales y los laboratorios ciudadanos.

\section{Referencias bibliográficas}

Abu-Shanab, E. A. (2015). Reengineering the open government concept: An empirical support for a proposed model. Government Information Quarterly, 32(4), 453-463. https://doi.org/10.1016/j.giq.2015.07.002 
Attard, J., Orlandi, F, Scerri, S., \& Auer, S. (2015). A systematic review of open government data initiatives. Government Information Quarterly, 32(4), 399-418. https://doi.org/10.1016/j.giq.2015.07.006

Bvuma, S., \& Joseph, B. K. (2019). Empowering Communities and Improving Public Services Through Open Data: South African Local Government Perspective. In Governance Models for Creating Public Value in Open Data Initiatives (pp. 141160). Springer, Cham.

Campos-Domínguez, E., \& García Orosa, B. (2018). Comunicación algorítmica en los partidos políticos: automatización de producción y circulación de mensajes. El Profesional de La Información, 27(4), 769-777.

Centro de Investigaciones Sociológicas (2019). Barómetro de septiembre 2019. Recuperado de: http://datos.cis.es/pdf/ Es3261marMT_A.pdf (10/10/2019)

Cotino, L. (2014) La nueva ley de transparencia y acceso a la información, Anuario Facultad de Derecho de la Universidad de Alcalá, VII, pp. 241-256.

Dader, J. L., Cheng, L., Campos, E., Quintana, N., \& Vizcaíno-Laorga, R. (2014). The Spanish Political Party Websites in Electoral Campaigning. Similar Trend since 2008 thru 2011. Trípodos, (34), 115-152.

Dader, J.L.; Cheng, L. (2011). “Análisis cuantitativo y cualitativo de las webs de partidos”. En: Sampedro, V. (coord.) Cibercampaña, cauces y diques para la participación. Las elecciones generales de 2008 y su proyección tecno-política. Madrid: Editorial Complutense. Libro Electrónico, p. 130-143.

Fomin, V. V, Pedersen, M. K., De Vries, H. J., \& Pedersen, M. K. (2008). Open Standards and Government Policy: Results of a Delphi Survey. Communications of the Association for Information Systems 22(25), 459-484

Gavilanes, M.A., Andrés, A. y Belomonte, E. (2016). ¿Cómo se financian los partidos políticos? Civio. Recuperado de: https://civio.es/el-boe-nuestro-de-cada-dia/2016/11/14/como-se-financian-los-partidos-politicos/ (9/10/2019).

Gobierno de España (2019) IV Plan de Gobierno abierto. Portal de Transparencia. Información disponible en: https:// transparencia.gob.es/transparencia/transparencia_Home/index/Gobierno-abierto.html (10/10/2019).

Grimmelikhuijsen, S. (2012). Transparency and Trust. An experimental study off online disclosure and trust in government. Utrecht University Repository.

Janssen, M., Charalabidis, Y., \& Zuiderwijk, A. (2012). Benefits, Adoption Barriers and Myths of Open Data and Open Government. Information Systems Management, 29(4), 258-268. https://doi.org/10.1080/10580530.2012.716740

Larrondo-Ureta, A. (2016). Comunicación organizacional ante el reto de la estrategia multiplataforma y 2.0: la experiencia de los partidos políticos en el País Vasco. El Profesional de La Información, 25(1), 114-123. http://doi.org/10.3145/ epi.2016.ene.11

Lathrop, D., y Ruma, L. (2010). Open government: Collaboration, transparency, and participation in practice. O'Reilly Media, Inc.

McDermott, P. (2010). Building open government. Government Information Quarterly, 27(4), 401-413. 
Meijer, A. J., Curtin, D., \& Hillebrandt, M. (2012). Open government: Connecting vision and voice. International Review of Administrative Sciences, 78(1), 10-29. https://doi.org/10.1177/0020852311429533

Meijer, A. J., Lips, M., \& Chen, K. (2019). Open Governance: A New Paradigm for Understanding Urban Governance in an Information Age. Frontiers in Sustainable Cities, 1(August), 1-9. https://doi.org/10.3389/frsc.2019.00003

Meijer, AJ, Curtin, D, Hillebrandt, M (2012) Open government: Connecting vision and voice. International Review of Administrative Sciences 78(1): 10-29.

Pérez, J. A., Peña, S., Genaut, A., Iturregui, L., \& Mendiguren, T. (2013). Comunicación política e Internet: Estrategias online de los partidos políticos vascos en las elecciones autonómicas de 2009. Mediatika, 14, 125-150.

Porlezza, C., \& Splendore, S. (2019). From Open Journalism to Closed Data: Data Journalism in Italy. Digital Journalism, $1-23$.

Rubio, R. (2011). Nuevas tecnologías y transparencia parlamentaria. Cuaderno Evoca, (4), pp 2-7.

Ruijer, E., Détienne, F., Baker, M., Groff, J., \& Meijer, A. J. (2019). The Politics of Open Government Data: Understanding Organizational Responses to Pressure for More Transparency. The American Review of Public Administration, 0275074019888065.

Ruvalcaba-Gomez, E. A., Criado, J. I., \& Gil-Garcia, J. R. (2018). Discussing open government as a concept: A comparison between the perceptions of public managers and current academic debate. ACM International Conference Proceeding Series. https://doi.org/10.1145/3209281.3209320

Ruvalcaba-Gomez, E. A., Sandoval-Almazan, R., Ignacio Criado, J., \& Valle-Cruz, D. (2019). Understanding the perspectives of open government: Exploratory factor analysis in Spain and Mexico. ACM International Conference Proceeding Series, (507), 507-508. https://doi.org/10.1145/3325112.3328216

Sierra Rodríguez, J. (2018). Mediciones y premios de transparencia. Revista Española de la Transparencia 7. Segundo Semestre 2018, pp. 71-97.

Vaccari, C. (2011) A tale of two e-parties: Candidate websites in the 2008 US presidential primaries, Party Politics, 19(1), pp. 19-40. doi: 10.1177/1354068810391287.

Wijnhoven, F, Ehrenhard, M, Kuhn, J (2015) Open government objectives and participation motivations. Government Information Quarterly 32(1): 30-42

Wirtz, B. W., Weyerer, J. C., \& Rösch, M. (2019). Open government and citizen participation: an empirical analysis of citizen expectancy towards open government data. International Review of Administrative Sciences, 85(3), 566-586. https://doi. org/10.1177/0020852317719996 


\section{Anexos}

\section{Participantes en el estudio Delphi}

\begin{tabular}{|c|c|}
\hline Nombre del participante & Perfil profesional \\
\hline Antoni Gutiérrez-Rubí & $\begin{array}{l}\text { Asesor internacional en comunicación política, institucional y empresarial. Director de } \\
\text { Ideograma }\end{array}$ \\
\hline Beatriz Martín Isidoro & $\begin{array}{l}\text { Doctora en Periodismo en la Universidad Complutense de Madrid y elaboradora de informes } \\
\text { de transparencia de los partidos políticos de la Fundación Compromiso y Transparencia }\end{array}$ \\
\hline David Rey Jordan & $\begin{array}{l}\text { Responsable Técnico Planificación (Universidad Pablo de Olavide); Profesor colaborador en } \\
\text { materia de transparencia e innovación pública en Instituto Nacional de Administraciones } \\
\text { Públicas, Universidad Internacional Menéndez Pelayo y Universidad Pablo de Olavide; co- } \\
\text { fundador Asociación Ciudadana OpenKratio. }\end{array}$ \\
\hline Elisa de la Nuez Sánchez-Cascado & Abogada del Estado. Secretaria general de la Fundación Hay Derecho. \\
\hline Enrique Cebrián & Profesor de Derecho Constitucional en la Universidad de Zaragoza. \\
\hline Francisco Delgado Morales & Presidente de la Asociación Española de Acreditación de la Transparencia. \\
\hline Jorge Resina & Profesor de Ciencia Política y de la Administración en la Universidad Complutense de Madrid \\
\hline José Luis Dader & Catedrático en Periodismo en la Universidad Complutense de Madrid \\
\hline José Manuel Sánchez Duarte & Profesor de Ciencia Política y Gestión Pública en la Universidad Rey Juan Carlos \\
\hline $\begin{array}{l}\text { Manuel Sánchez de Diego } \\
\text { Fernández de la Riva }\end{array}$ & $\begin{array}{l}\text { Director del Máster Propio en Transparencia y Acceso a la Información Pública de la UCM y } \\
\text { fundador de la Coalición Pro Acceso. Profesor de Derecho Constitucional. }\end{array}$ \\
\hline Miguel Ángel Gonzalo & $\begin{array}{l}\text { Webmaster del Congreso de los Diputados. Profesor del Máster en Transparencia y Acceso a la } \\
\text { Información Pública de la UCM. }\end{array}$ \\
\hline Nagore de los Ríos & $\begin{array}{l}\text { CEO en Nagoredelosrios.com y en OutreachTool SL. Data Driven Communication Senior } \\
\text { Consultant. }\end{array}$ \\
\hline
\end{tabular}

